\title{
Integration of Different “-omics” Technologies Identifies Inhibition of the IGF1R-Akt-mTOR Signaling Cascade Involved in the Cytotoxic Effect of Shikonin against Leukemia Cells
}

\author{
Benjamin Wiench,, ${ }^{1}$ Yet-Ran Chen, ${ }^{2}$ Malte Paulsen, ${ }^{3}$ Rebecca Hamm, ${ }^{1}$ Sven Schröder, \\ Ning-Sun Yang, ${ }^{2}$ and Thomas Efferth ${ }^{1}$ \\ ${ }^{1}$ Institute of Pharmacy and Biochemistry, Department of Pharmaceutical Biology, Johannes Gutenberg University, \\ Staudinger Weg 5, 55128 Mainz, Germany \\ ${ }^{2}$ Agricultural Biotechnology Research Center, Academia Sinica, 115 Taipei, Taiwan \\ ${ }^{3}$ Cytometry Core Facility, Institute of Molecular Biology, Ackermannweg 4, 55128 Mainz, Germany \\ ${ }^{4}$ HanseMerkur Center for Traditional Chinese Medicine at the University Medical Center Eppendorf, 20246 Hamburg, Germany
}

Correspondence should be addressed to Thomas Efferth; efferth@uni-mainz.de

Received 18 March 2013; Accepted 7 May 2013

Academic Editor: Sookyung Lee

\begin{abstract}
Copyright (C) 2013 Benjamin Wiench et al. This is an open access article distributed under the Creative Commons Attribution License, which permits unrestricted use, distribution, and reproduction in any medium, provided the original work is properly cited.

Hematological malignancies frequently have a poor prognosis and often remain incurable. Drug resistance, severe side effects, and relapse are major problems of currently used drugs, and new candidate compounds are required for improvement of therapy success. The naphthoquinone shikonin derived from the Chinese medicinal herb, Lithospermum erythrorhizon, is a promising candidate for the next generation of chemotherapy. The basal cellular mechanism of shikonin is the direct targeting of mitochondria. Cytotoxicity screenings showed that the compound is particularly effective against leukemia cells suggesting an additional cellular mechanism. mRNA and miRNA microarrays were used to analyze changes in gene expression in leukemia cells after shikonin treatment and combined with stable-isotope dimethyl labeling for quantitative proteomics. The integration of bioinformatics and the three "-omics" assays showed that the PI3K-Akt-mTOR pathway was affected by shikonin. Deregulations of this pathway are frequently associated with cancerogenesis, especially in a wide range of hematological malignancies. The effect on the PI3K-Akt-mTOR axis was validated by demonstrating a decreased phosphorylation of Akt and a direct inhibition of the IGF1R kinase activity after shikonin treatment. Our results indicate that inhibiting the IGF1R-Akt-mTOR signaling cascade is a new cellular mechanism of shikonin strengthening its potential for the treatment of hematological malignancies.
\end{abstract}

\section{Introduction}

Hematological malignancies comprise different types of cancers that affect blood cells, bone marrow, or lymph nodes. According to the National Cancer Institute, leukemia, lymphoma, and myeloma accounted for $9 \%$ of all deaths from cancer in the United States in 2011. In recent years improvements in radio therapy and new therapeutics including imatinib [1], rituximab [2], and lenalidomide [3] clearly improved the response and survival rate of these diseases [4]. Despite this considerable advances in chemotherapy, drug resistance and relapse remain major problems and several hematological malignancies remain incurable with standard treatments $[4,5]$. This lack of cures requires novel targeted and less toxic therapies, and new candidate compounds need to be tested for the improvement of therapy success.

The naphthoquinone pigment shikonin is the most important pharmacologically active substance in the dried root of Lithospermum erythrorhizon. In traditional Chinese medicine (TCM) root extracts of Lithospermum erythrorhi$z o n$ have been used to treat macular eruption, measles, sorethroat, carbuncles, and burns [6]. We recently showed that the natural compound shikonin has a strong cytotoxic effect against different cancer cell lines, especially sensitive and 
resistant leukemia cells. Shikonin directly targets mitochondria of cancer cells causing an overproduction of reactive oxygen species (ROS), mitochondrial dysfunction, and ultimately apoptosis [7]. However, the question why shikonin is particularly effective against leukemia and lymphoma cells remains unresolved.

In the present study, we investigated the effect of shikonin on the myeloid leukemia cell line U937 by the integration of different quantitative "-omics" technologies, combining high-throughput techniques as a promising tool for elucidating molecular mechanisms of new drugs in a fast and precise manner [8]. The integration of genomic and pharmacological analysis significantly accelerates the identification of cancer-specific synthetic lethal targets [9]. We analyzed the mRNA, miRNA, and protein expression in U937 cells after shikonin treatment and integrated the results using a bioinformatic approach. Thereby, it was possible to identify cellular functions and signaling pathways strongly deregulated after shikonin treatment. The data obtained from the proteomic and transcriptomic studies confirmed previous findings indicating that shikonin has strong effects on cell proliferation, cell cycle progression, cellular movement, and DNA integrity of cancer cells [7]. Interestingly, our findings indicated that one of the most affected signaling pathways in U937 leukemia cells was the phosphatidylinositol 3-kinase (PI3K)-Akt-mammalian target of rapamycin (mTOR) cascade. Hence, we proposed that an inhibition of this signaling network is a reason for the strong activity of shikonin against leukemia cells. We validated the effect of shikonin on the PI3K-AktmTOR pathway by demonstrating a decreased phosphorylation and activation of Akt after shikonin treatment using phospho-specific antibodies and flow cytometric analysis. In addition, kinase activity tests revealed that shikonin inhibits the kinase activity of the insulin-like growth factor 1 receptor (IGF1R), which is an important trigger of the PI3K-AktmTOR signaling cascade.

Targeting of PI3K-Akt-mTOR signaling became an attractive therapeutic strategy for cancer chemotherapy over the last few years $[10,11]$. The signaling pathway plays a central role in cellular growth and survival through the regulation of protein synthesis and ribosomal protein translation [12]. Deregulations of mTOR signaling are associated with tumorgenesis, angiogenesis, tumor growth, and metastasis [10, 13]. The mTOR signaling pathway has been found to be frequently deregulated, especially in a wide range of hematological malignancies [14]. The signaling cascade is activated by receptor tyrosine kinases (RTKs, e.g., IGF1R and epidermal growth factor receptor (EGFR)), integrins, and cytokine receptors coupling external signals from growth factors, cytokines and the availability of nutrients to cell growth and proliferation [15]. After binding of the corresponding ligands, the RTKs activate PI3K, which in turn causes the phosphorylation of Akt. Activated Akt inhibits the heterodimeric complex of tuberous sclerosis proteins 1 and 2 (TSC1/2) that negatively regulates the mammalian target of rapamycin complex 1 (mTORC1) [16]. This complex is a centerpiece of the signaling cascade that controls protein synthesis by phosphorylation of different effector proteins, for example, the S6 kinase 1 (S6K1) and the 4E-binding protein 1 (4E-BP1) [17]. Much

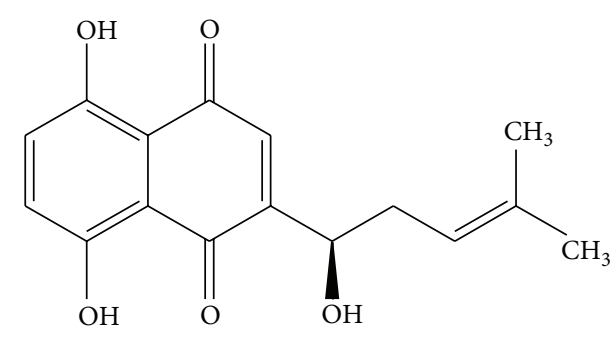

(a)

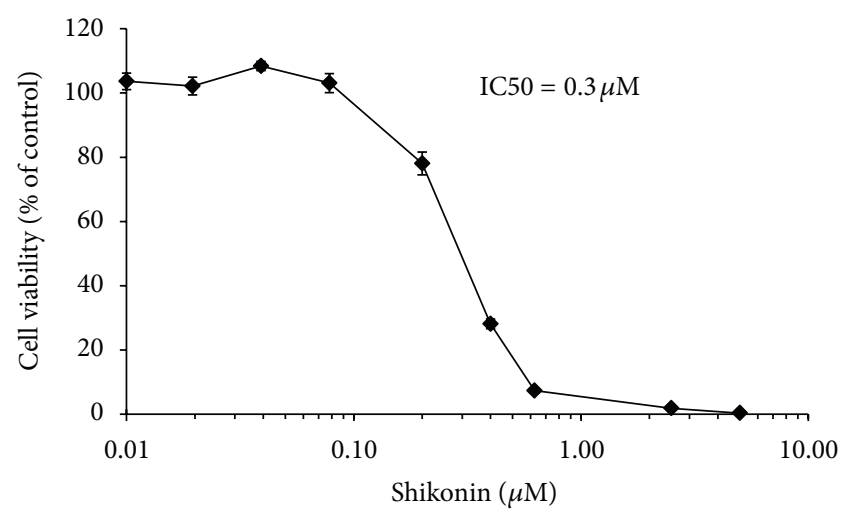

(b)

FIgURE 1: Cytotoxic effect of shikonin against U937 leukemia cells. (a) Chemical structure of shikonin. (b) Dose response curves of U937 cells after treatment with different concentrations of shikonin. Resazurin reduction assays were performed to determine dose response curve. Viability of U937 cells is represented by mean \pm SEM of three independent experiments, and it is expressed as percentage survival of control.

less is known about the second mTOR complex mTORC2. This complex responds to growth factors and regulates cell survival and metabolism, as well as the cytoskeleton [17]. Currently used drugs targeting this pathway are rapamycin and its derivatives (rapalogs) that directly target the mTORC1 complex $[18,19]$. One weak point of these drugs is a resistance mechanism of cancer cells, which leads to an upregulation of IGF1R after mTORC1 inhibition [20-22]. This feedback mechanism causes an activation of the PIK3K-Akt-mTOR signaling cascade after initial inhibition resulting in only modest anticancer effects of rapalogs [14].

Ultimately, our results suggest that inhibition of IGF1RAkt-mTOR signaling plays a key role in the cytotoxic effect of shikonin against U937 leukemia cells. Since this signaling network is frequently deregulated in hematological malignancies, shikonin is a promising candidate for the next generation of chemotherapy against these diseases.

\section{Results}

2.1. Cytotoxic Effect of Shikonin on U937 Leukemia Cells. The cytotoxic effect of shikonin against U937 leukemia cells was analyzed by resazurin reduction assay. The shikonin dose response curve was calculated after a $24 \mathrm{~h}$ treatment of subconfluent U937 cells (Figure 1). Shikonin inhibited 
U937 proliferation reproducibly by $50 \%$ at a concentration of $0.3 \mu \mathrm{M}$, and this concentration was subsequently used in experiments for gene expression profiling and stable-isotope dimethyl labeling for quantitative proteomics.

\subsection{Omics Data Reveals New Insights into Cellular Mecha-} nisms of Shikonin. To get deeper insights into the molecular modes of action of shikonin in U937 cells, three "-omics" assays were performed (mRNA microarray, miRNA microarray, and stable-isotope dimethyl labeling for quantitative proteomics) quantifying transcriptomic and proteomic changes after shikonin treatment. The Venn diagram shows the number of deregulated molecules in each assay and the number of predicted mRNA targets of deregulated miRNAs (Figure 2). The intersections of the diagram indicate the number of corresponding molecules deregulated in different assays independently from the direction of their deregulated expression. The overlap of deregulated genes and proteins comprises 88 molecules (19\%). Filtered to cases where the corresponding molecules are expressed in the same biological direction, for example up-regulated mRNA levels causing increased protein expression, the total overlap is limited to 52 molecules (11\%). The top deregulated molecules are listed in Table 1.

Since each of the three "-omics" assays resulted in a plethora of information about shikonin's cellular mechanisms, we decided to evaluate the datasets in a comparative approach by extracting the most significant results. The data of the single assays were matched using the Ingenuity Pathway Analysis (IPA) comparative analysis tool, revealing cellular functions deregulated by shikonin treatment on the transcriptome as well as on the proteome (Figure 3(a)). The data confirms previous findings indicating that shikonin has strong effects on cell proliferation, cell cycle progression, cellular movement, and DNA integrity of cancer cells. Interestingly, shikonin also affects the posttranscriptional modification of RNA and disturbs cell-to-cell signaling and interaction. The data of the three "-omics" assays were compiled to one data set, and a pathway analysis was performed using IPA. This reanalysis identified a signaling network around the PI3K-Akt-mTOR axis, which was strongly affected by shikonin treatment (Figure 3(b)).

\subsection{Effect of Shikonin on the PI3K-mTOR Signaling Cascade.} We analyzed the PI3K-Akt-mTOR signaling pathway by a close examination of the upstream marker p-Akt and the downstream marker p-ribosomal protein S6 (pRiboS6) by flow cytometry. Samples with and without shikonin treatment were stained with directly conjugated antibodies against p-Akt and pRiboS6. Shikonin significantly inhibits the phosphorylation of Akt, while the phosphorylation of RiboS6 remained almost unchanged (Figure 4). This result indicates an effect of shikonin upstream of the PI3K-mTOR signaling cascade.

2.4. Virtual Screening. To identify possible targets of shikonin in the PI3K-mTOR pathway, we conducted a virtual screening approach. Binding energies of shikonin and key proteins

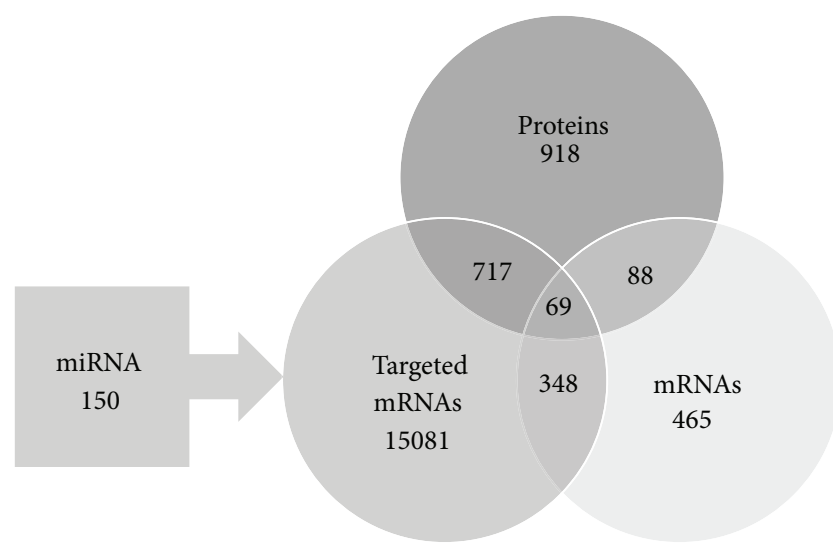

FIGURE 2: Results and relations of mRNA microarray, miRNA microarray, and stable-isotope dimethyl labeling for quantitative proteomics. Only molecules from the omics datasets that met the expression fold change cutoff of $\geq \pm 1.2$ are shown in this diagram. The intersections of the Venn diagram indicate the number of corresponding molecules deregulated in different assays independently from the direction of their deregulated expression.

of the signaling cascade-with available crystal structureswere calculated using the AutoDock Vina tool [23]. Proteins involved in the PI3K-mTOR pathway were selected according to a recent publication by Laplante and Sabatini [17]. Table 2 summarizes the ranking of the predicted target proteins of shikonin. Within the top potential binding partners, we found the receptor tyrosine kinases EGFR and IGF1R and the Serine/threonine-protein kinase Sgk1 (SGK1). Since the results of the Akt/RiboS6 phosphorylation by FACS showed no effect of shikonin on the phosphorylation status of ribosomal S6 protein, a target of SGK1, we did not further investigate the kinase as target of shikonin. EGFR and IGF1R are both important transmembrane receptors that trigger the PI3K-mTOR signaling cascade after binding to their respective growth factors like EGF, TGF $\alpha$, and IGF. Previous studies showed that shikonin inhibits EGFR phosphorylation and modulates the EGFR signaling cascade [24], and we concentrated on the IGF1R for our further analysis of shikonin action, due to its known evolvement in drug evasion.

2.5. Shikonin Inhibits IGF1R kinase Activity. Since IGF1R showed a very high binding activity towards shikonin in the virtual screening experiment, we tested the inhibitory effect of shikonin on this kinase using a radiometric protein kinase activity assay. The dose response curve of shikonin on the IGF1R activity clearly indicates a dose-dependent inhibition of the kinase after shikonin application (Figure 5). An IC50 concentration of $2.6 \mu \mathrm{M}$ was calculated for shikonin on the purified IGF1R kinase activity by nonlinear regression using Prism 5.04 (Graphpad, CA, USA). This IC50 of shikonin on the IGF1R kinase is very similar to the IC50 of about $3 \mu \mathrm{M}$ observed for U937 cells suggesting that shikonin-besides inducing mitochondrial dysfunction-also exerts additional anticancer activities specifically by inhibiting IGF1R. 


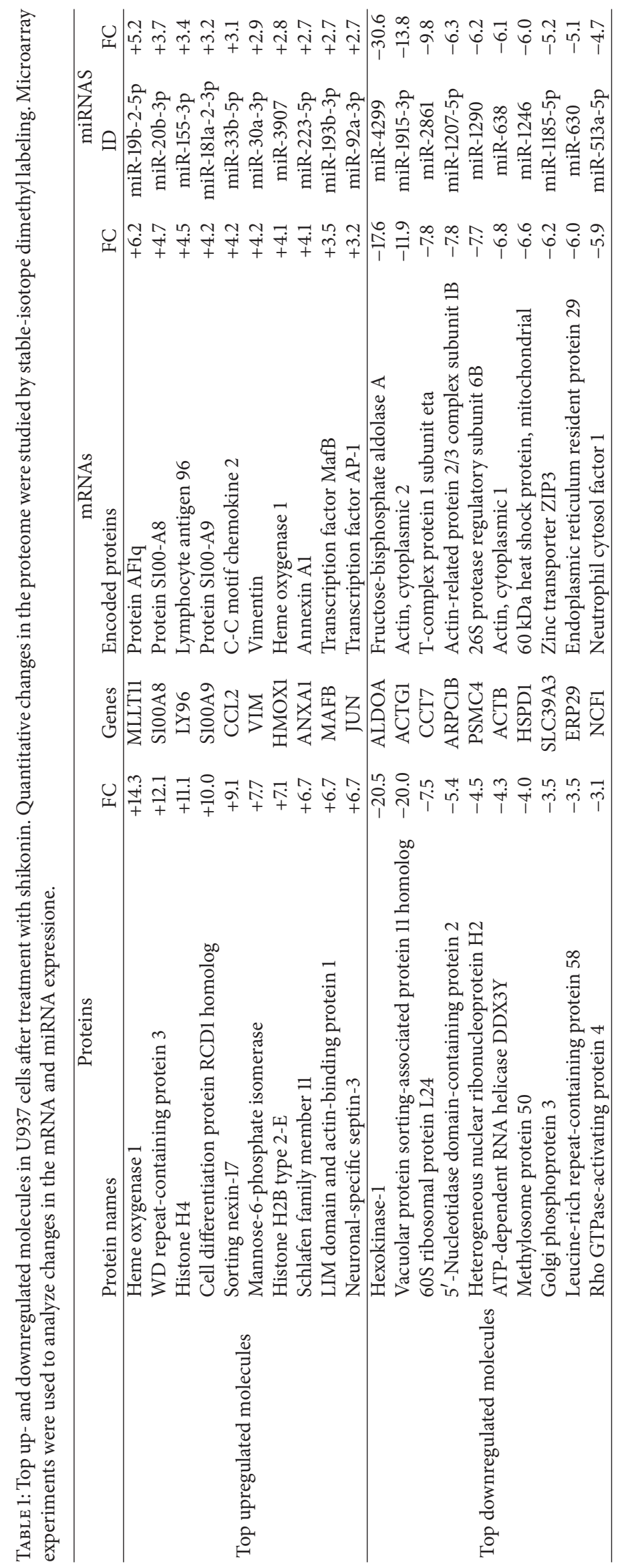




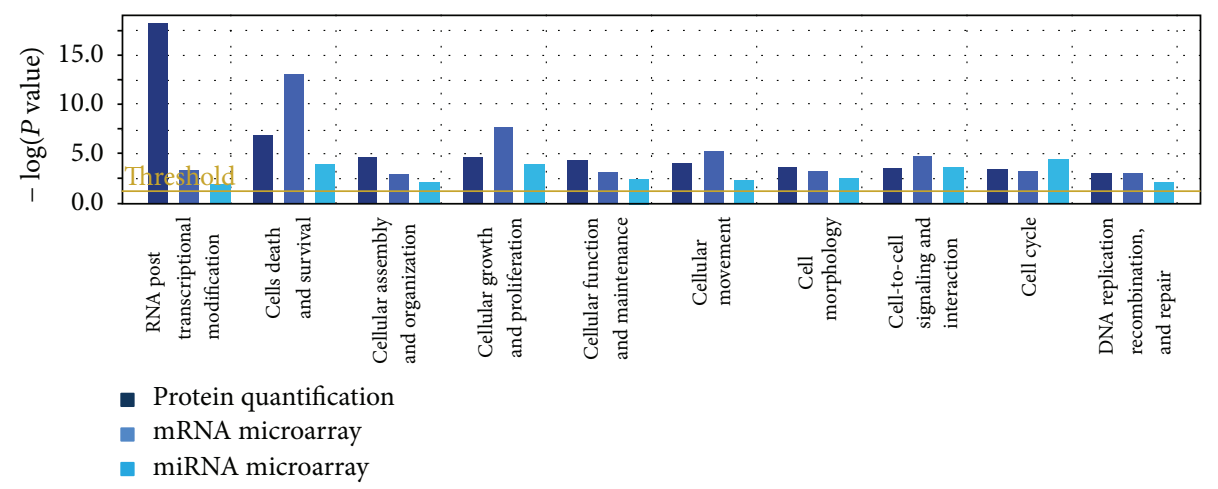

(a)

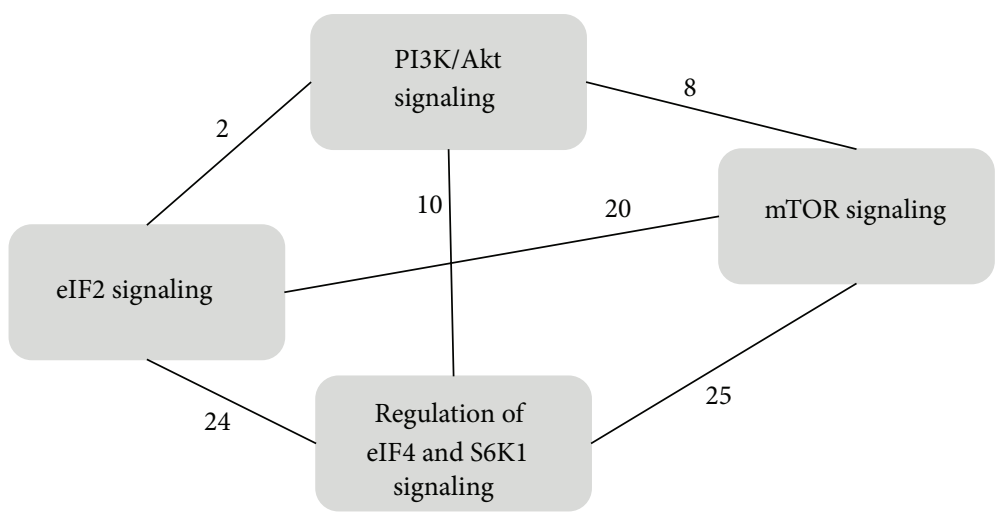

(b)

FIGURE 3: Complementarity of various “-omics” technologies provides a system-level understanding of shikonin's effects in U937 cells. (a) Comparison analysis of molecular and cellular functions affected by shikonin in three "-omics" assays. The bar graph displays only functions disturbed in all three assays. Right-tailed Fisher's exact test was used to calculate a $P$ value determining the probability that each biological function assigned to the datasets is due to chance alone. (b) Overlapping signaling pathways deregulated after shikonin treatment. Numbers of identical molecules deregulated in overlapping pathways are shown.

\section{Discussion}

The combination of "-omics" data is a powerful tool to investigate the cellular effects and mechanisms of small molecules [25]. In the present study, we used this experimental approach to show that the natural naphthoquinone shikonin strongly deregulates the IGF1R-Akt-mTOR signaling cascade in U937 myeloid leukemia cells. Three different "-omics" assay indicated that the signaling pathway is disturbed at transcriptomic and proteomic level. Functional validation studies showed that shikonin indeed inhibits two central signaling nodes of the pathway: the kinase activity of IGF1R (a central receptor of the signaling cascade) was inhibited, and in addition the phosphorylation of Akt was significantly decreased upon shikonin application.

The PI3K-Akt-mTOR cascade is the predominant signal transduction pathway after IGF1R activation [15]. Our results are consistent with previous findings indicating that an inhibition of IGF1R causes a decreased phosphorylation and activation of Akt [26]. The reduced Akt phosphorylation in turn causes a decreased activation of the mTOR complexes (mTORC1/2) [12]. mTORC1 regulates cellular protein synthesis by phosphorylation of $\mathrm{S} 6 \mathrm{~K} 1$ and $4 \mathrm{E}-\mathrm{BP} 1$, and
mTORC2 controls cellular growth and survival through the phosphorylation of many AGC kinases including Akt, SGK1, and PKC- $\alpha$ [17]. It was shown that inhibition of critical signaling nodes of this pathway induces cell cycle arrest and apoptosis in leukemia cells [27]. These findings corroborate our results indicating that the cytotoxic effect of shikonin against leukemia cells is reinforced by a direct inhibition of IGF1R and a deregulation of the IGF1R-Akt-mTOR signaling cascade.

The signaling network around the mTOR kinase has been shown to be frequently deregulated in a wide range of hematological malignancies, especially in different types of leukemia [14]. For example, in acute myelogenous leukemia (AML), the mTORC1-signaling pathway is constitutively activated in almost $100 \%$ of all patients [28, 29]. Blocking this pathway could be an effective new treatment strategy for leukemia and other hematological malignancies. At the moment, rapamycin and its derivatives (rapalogs), for example, RAD001 (everolimus), CCI-779 (temsirolimus), and AP23573 (deforolimus), are used to partially inhibit the signaling pathway by directly binding the mTORC1 complex $[18,19]$. However, results of clinical trials were mostly disappointing and showed only modest anticancer effects 


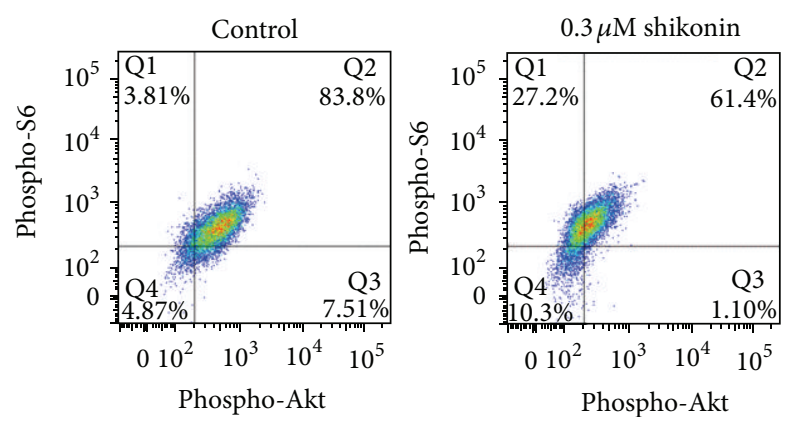

(a)

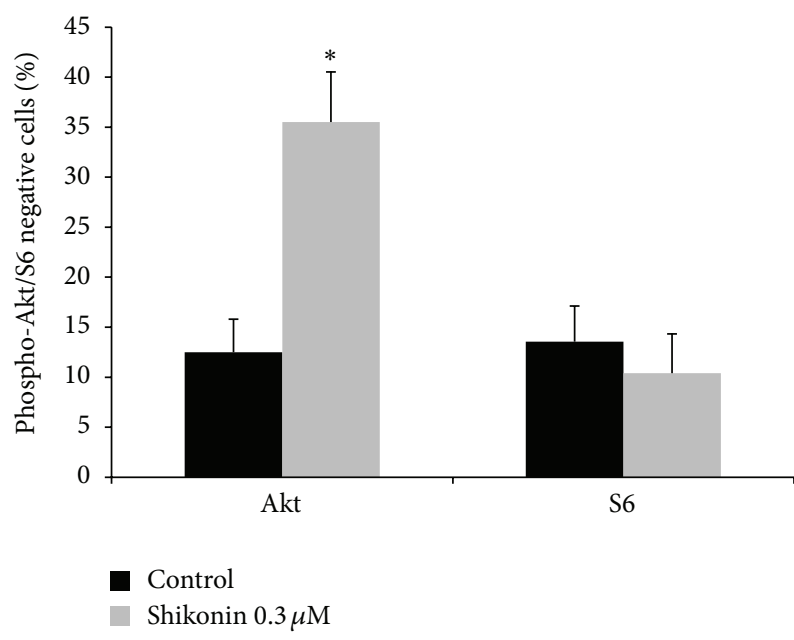

(b)

FIGURE 4: Effect of shikonin on the PI3K-mTOR signaling cascade. (a) U937 cells were treated with $0.3 \mu \mathrm{M}$ shikonin for $24 \mathrm{~h}$ and subsequently stained with phospho-specific antibodies against pAkt and p-ribosomal protein S6. Shikonin treatment significantly decreased the amount of phosphorylated Akt (left shift), but no effect on the phosphorylation status of ribosomal protein S6 was detected. (b) Statistical quantification of p-Akt or p-ribosomal protein S6 negative cells after shikonin treatment. Data points represent mean $\pm \mathrm{SD}$ of at least three independent experiments. ( ${ }^{*}$ Significantly different according to Student's $t$-test, $P<0.05$ ).

of these drugs [14]. A combination of rapalogs with other targeted molecules was more successful, and clinical trials have shown that rapalogs synergize with different conventional chemotherapeutics to overcome resistance [14, 30, 31]. Recent findings showed that a positive feedback loop causes the rapamycin-resistance phenotype: mTOR inhibition by rapalogs leads to an upregulation of IGF1R signaling, which in turn activates the PI3K-Akt-mTOR cascade again [20-22]. Thus, combining an mTOR inhibitor and an IGF-1R inhibitor may be an appropriate strategy to enhance mTOR-targeted anticancer therapy [22]. Since shikonin strongly deregulates the mTOR signaling pathway and in addition directly inhibits the kinase activity of IGF1R, it is a promising candidate for a cotreatment with rapalogs.

Our results are consistent with recent findings indicating that shikonin also modulates cell proliferation by inhibiting EGFR signaling [24]. EGFR is a further RTK that triggers the
TABLE 2: Calculation of binding energies of shikonin and key proteins of the IGF1R-Akt-mTOR signaling pathway. Virtual screening was performed using the AutoDock Vina software.

\begin{tabular}{|c|c|c|}
\hline Symbol & Description & $\begin{array}{c}\text { Binding energy } \\
{[\mathrm{kcal} / \mathrm{mol}]}\end{array}$ \\
\hline EGFR & Epidermal growth factor receptor & -8.8 \\
\hline SGK1 & Serine/threonine-protein kinase Sgk1 & -8.8 \\
\hline IGF1R & Insulin-like growth factor 1 receptor & -8.6 \\
\hline GSK3B & Glycogen synthase kinase- 3 beta & -8.5 \\
\hline 4E-BP1 & $\begin{array}{l}\text { Eukaryotic translation initiation factor } \\
\text { 4E-binding protein } 1\end{array}$ & -8.5 \\
\hline S6K1 & Ribosomal protein S6 kinase beta-1 & -8.4 \\
\hline PKCA & Protein kinase $\mathrm{C}$ alpha type & -8.4 \\
\hline PIK3C3 & $\begin{array}{l}\text { Phosphatidylinositol 3-kinase catalytic } \\
\text { subunit type } 3\end{array}$ & -8.3 \\
\hline PDK1 & $\begin{array}{l}\text { 3-phosphoinositide-dependent protein } \\
\text { kinase } 1\end{array}$ & -8.3 \\
\hline eIF4E & $\begin{array}{l}\text { Eukaryotic translation initiation factor } \\
4 \mathrm{E}\end{array}$ & -8.3 \\
\hline RND3 & $\begin{array}{l}\text { Rho-related GTP-binding protein } \\
\text { RhoE }\end{array}$ & -8.2 \\
\hline AKT2 & $\begin{array}{l}\text { RAC-beta serine/threonine-protein } \\
\text { kinase }\end{array}$ & -8.1 \\
\hline RSK1 & Ribosomal protein S6 kinase alpha-1 & -8.1 \\
\hline Rheb & GTP-binding protein Rheb & -7.9 \\
\hline AMPK2 & $\begin{array}{l}5^{\prime} \text {-AMP-activated protein kinase } \\
\text { catalytic subunit alpha- } 2\end{array}$ & -7.9 \\
\hline RHOA & Transforming protein RhoA & -7.9 \\
\hline AKT1 & $\begin{array}{l}\text { RAC-alpha serine/threonine-protein } \\
\text { kinase }\end{array}$ & -7.8 \\
\hline RRAGD & Ras-related GTP-binding protein D & -7.8 \\
\hline mTOR & $\begin{array}{l}\text { Serine/threonine-protein kinase } \\
\text { mTOR }\end{array}$ & -7.5 \\
\hline RHOC & $\begin{array}{l}\text { Rho-related GTP-binding protein } \\
\text { RhoC }\end{array}$ & -7.3 \\
\hline RHOB & $\begin{array}{l}\text { Rho-related GTP-binding protein } \\
\text { RhoB }\end{array}$ & -7.2 \\
\hline ERK1 & Extracellular signal-regulated kinase 1 & -7.1 \\
\hline RHOD & $\begin{array}{l}\text { Rho-related GTP-binding protein } \\
\text { RhoD }\end{array}$ & -6.9 \\
\hline IRS1 & Insulin receptor substrate 1 & -6.4 \\
\hline IKKB & $\begin{array}{l}\text { Inhibitor of nuclear factor kappa-B } \\
\text { kinase subunit beta }\end{array}$ & -5.1 \\
\hline
\end{tabular}

PI3K-Akt-mTOR cascade. Results showed that simultaneous targeting of EGFR and mTOR inhibits the growth of cancer cells [32]. An additional inhibition of EGFR signaling makes shikonin even more valuable for targeting the PI3K/mTOR cascade since a second important starting point of the pathway is eliminated.

Besides the deregulation of the PI3K-Akt-mTOR signaling cascade, we recently showed that shikonin directly targets the mitochondria of cancer cells and thereby triggers apoptosis [7]. Mitochondria targeting drugs were suggested to synergize with the common and clinically established 


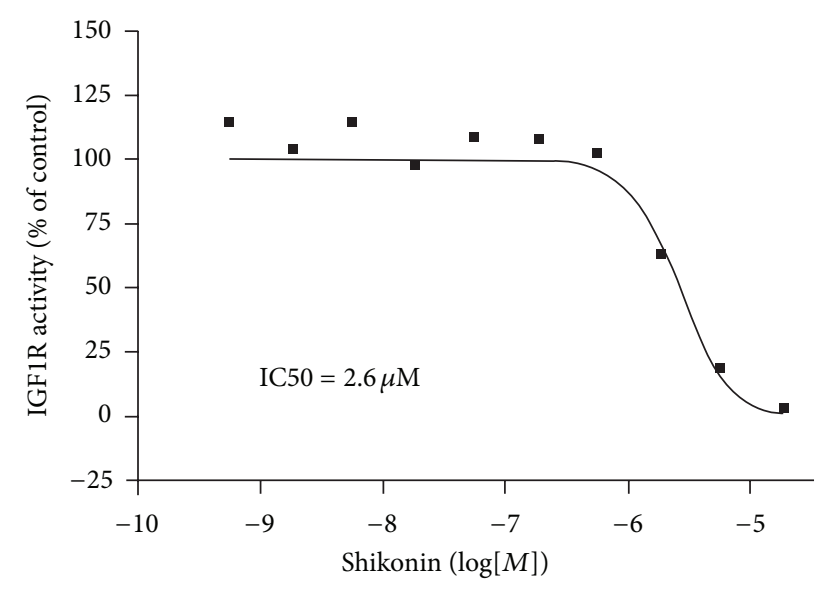

Figure 5: Dose response curve of shikonin in an IGF1-R kinase assay. A radiometric protein kinase assay (33PanQinase Activity Assay) was used for measuring the kinase activity of IGF1R. IC50 values were calculated by nonlinear regression using Prism 5.04 (Graphpad, CA, USA).

antileukemic drug arsenite trioxide [33]. This makes shikonin even more interesting for the treatment of leukemia. On the one hand, it inhibits the PI3K-mTOR signaling cascade, which is frequently deregulated in leukemia, and in addition it is directly targeting mitochondria of cancer cells, which makes it to a promising candidate for a combined treatment with established antileukemic drugs.

Ultimately, the direct targeting of mitochondria and the simultaneous deregulation of the IGF1R-Akt-mTOR signaling cascade make shikonin a promising compound for the treatment of hematological malignancies.

\section{Materials and Methods}

4.1. Chemicals. Shikonin was purchased from Enzo Life Sciences (Lausen, Switzerland) and a $50 \mathrm{mM}$ stock solution was prepared by dissolving it in DMSO. Triethylammonium bicarbonate (TEABC), sodium cyanoborohydride, ammonium hydroxide, formaldehyde (37\% solution in $\mathrm{H}_{2} \mathrm{O}$ ), formaldehyde- $13 \mathrm{C}, \mathrm{d} 2\left(20 \%\right.$ solution in $\left.\mathrm{D}_{2} \mathrm{O}\right)$, tris (2carboxyethyl) phosphine hydrochloride (TCEP), ammonium persulfate (APS), methyl methanethiosulfonate (MMTS), Triton X-100 (TX-100), 4-(2-hydroxyethyl) piperazine1-ethanesulfonic acid (HEPES), sodium bicarbonate, $\mathrm{N}, \mathrm{N}, \mathrm{N}^{\prime}, \mathrm{N}^{\prime}$-Tetramethylenediamine (TEMED), $40 \%$ acrylamide/bis-acrylamide (37.5:1) solution, trifluoroacetic acid (TFA), formic acid (FA), bovine serum albumin (BSA), and bovine beta casein were purchased from SigmaAldrich (St. Louis, MO). Ethanol, methanol, acetonitrile $(\mathrm{ACN})$, and sodium dodecyl sulfate (SDS) were purchased from J. T. Baker (Phillipsburg, NJ). Trypsin (modified, sequencing grade) was from Promega (Madison, WI). Deionized water $(18.1 \mathrm{M} \Omega \cdot \mathrm{cm}$ resistivity) from Milli-Q system (Millipore, Bedford, MA) was used throughout this work.
4.2. Cell Cultures. U937 cells were obtained from the German Cancer Research Center (DKFZ, Heidelberg, Germany). The original source of the cell line is the American Type Culture Collection (ATCC, USA). U937 cells were maintained in complete RPMI 1640 medium with $2 \mathrm{mM}$ L-glutamine (Invitrogen, Germany) supplemented with 10\% FBS (Invitrogen, Germany) and $1 \%$ penicillin $(100 \mathrm{U} / \mathrm{mL})$-streptomycin $(100 \mu \mathrm{g} / \mathrm{mL})$ (Invitrogen, Germany). Cells were maintained in a humidified environment at $37^{\circ} \mathrm{C}$ with $5 \% \mathrm{CO}_{2}$ and subcultured twice per week. All experiments were performed on cells in the logarithmic growth phase.

4.3. Resazurin Reduction Assay. Resazurin reduction assay [34] was performed to assess cytotoxicity of shikonin toward U937 cells. The assay is based on reduction of the indicator dye, resazurin, to the highly fluorescent resorufin by viable cells. Nonviable cells rapidly lose the metabolic capacity to reduce resazurin and thus produce no fluorescent signal. Briefly, aliquots of $2 \times 10^{4}$ U937 cells per well were seeded in $96-$ well plates in a total volume of $100 \mu \mathrm{L}$. Shikonin was immediately added in varying concentrations in an additional $100 \mu \mathrm{L}$ of culture medium to obtain a total volume of $200 \mu \mathrm{L} /$ well. After $24 \mathrm{~h}, 20 \mu \mathrm{L}$ resazurin (Sigma-Aldrich, Germany) $0.01 \% \mathrm{w} / \mathrm{v}$ in $\mathrm{ddH}_{2} \mathrm{O}$ was added to each well and the plates were incubated at $37^{\circ} \mathrm{C}$ for $4 \mathrm{~h}$. Fluorescence was measured on an Infinite M2000 Pro plate reader (Tecan, Germany) using an excitation wavelength of $544 \mathrm{~nm}$ and an emission wavelength of $590 \mathrm{~nm}$. Each assay was done at least two times, with six replicates each. The viability was evaluated based on a comparison with untreated cells. $\mathrm{IC}_{50}$ value represents the shikonin concentrations required to inhibit $50 \%$ of cell proliferation and was calculated from a calibration curve by linear regression using Microsoft Excel.

4.4. mRNA Microarray. Total RNA from U937 cells after $24 \mathrm{~h}$ of treatment with shikonin at $\mathrm{IC}_{50}$ concentration or DMSO solvent control was isolated using RNeasy Kit from Qiagen (Hilden, Germany) according to the manufacture's instruction. The quality of total RNA was checked by gel analysis using the total RNA Nano chip assay on an Agilent 2100 Bioanalyzer (Agilent Technologies $\mathrm{GmbH}$, Berlin, Germany). Only samples with RNA index values greater than 9.3 were selected for expression profiling. Microarray experiments were performed in duplicates for treated and untreated samples. Biotin-labeled cRNA samples for hybridization on Illumina Human Sentrix-HT12 Bead Chip arrays (Illumina, Inc.) were prepared according to Illumina's recommended sample labeling procedure based on the modified Eberwine protocol [35]. Biotin-16-UTP was purchased from Roche Applied Science (Penzberg, Germany). The cRNA was column purified with TotalPrep RNA Amplification Kit and eluted in $60 \mu \mathrm{L}$ of water. Quality of cRNA was controlled using the RNA Nano Chip Assay on an Agilent 2100 Bioanalyzer and spectrophotometrically quantified (NanoDrop). Hybridization was also performed according the manufacturer's recommendations. Microarray scanning was done using a Beadstation array scanner, setting adjusted to a scaling factor of 1 and PMT settings at 430 . 
Data was extracted for each bead individually, and outliers were removed when the MAD (median absolute deviation) was greater than 2.5. Data analysis was performed by using the quantile normalization algorithm without background subtraction, and differentially regulated genes were defined by calculating the standard deviation differences of a given probe in a one-by-one comparison of samples or groups. The expression data obtained was filtered with Chipster data analysis platform. These steps include filtering of genes by two times standard deviation of deregulated genes and a subsequent assessment of significance using empirical Bayes $t$-test $(P<0.05)$.

4.5. Real-Time Reverse Transcription PCR. The same RNA samples used in the microarray experiments were also used for RT-PCR experiments. Total RNA samples were converted to cDNA by reverse transcriptase (Invitrogen) with random hexamer primers. Quantification of cDNA was performed by real-time PCR using a Taq-polymerase master mix (Roche) containing the fluorescent dye SYBR Green (Biozol) and the CFX384 Real-Time PCR Detection System (Bio-Rad). The efficiency of all primer pairs used for real-time PCR expression was better than $90 \%$. PCR was performed with an initial denaturation at $95^{\circ} \mathrm{C}$ for $5 \mathrm{~min}$ followed by 50 cycles consisting of strand separation at $95^{\circ} \mathrm{C}$ for $30 \mathrm{~s}$ and annealing and extension at $60^{\circ} \mathrm{C}$ for $40 \mathrm{~s}$. After PCR product amplification, melting curves were computed. Expression levels were normalized to the transcription level of G6PD. All samples were run in triplicates.

4.6. miRNA Microarray. miRNA from U937 cells after $24 \mathrm{~h}$ of treatment with shikonin at $\mathrm{IC}_{50}$ concentration was isolated using miRNeasy Kit from Qiagen (Hilden, Germany) according to the manufacture's instruction. The quality of miRNA was checked by gel analysis using the Small RNA Nano chip assay on an Agilent 2100 Bioanalyzer (Agilent Technologies $\mathrm{GmbH}$, Germany). The miRNA microarray was performed at the Institute for Molecular Biology (IMB). Microarray experiments were performed in duplicates for treated and untreated samples. Human miRNA microarray chips $(8 \times 60 \mathrm{~K}$, Agilent Technologies $)$ were used. Probe labeling and hybridization were carried out following the miRNA microarray system with miRNA complete labeling and hyb kit protocol (Agilent Technologies). Briefly, extracted RNA was treated with phosphatase. Dephosphorylated RNA was fluorescently labeled by ligation of Cyanine 3-pCp molecules to the $3^{\prime}$ end of RNA molecules using Agilent's miRNA Complete Labeling and Hyb Kit. The labeled RNA was desalted and hybridized for 20 hours at $55^{\circ} \mathrm{C}$ and $20 \mathrm{rpm}$. Microarray slides were washed and scanned with Agilent Microarray Scanning system. Images were analyzed and data were extracted, background subtracted, and normalized using the standard procedures of Agilent Feature Extraction Software. The expression data obtained was filtered with Chipster data analysis platform. These steps include filtering of miRNAs by two times standard deviation of deregulated genes and a subsequent assessment of significance using empirical Bayes $t$-test $(P<0.05)$.
4.7. Proteomics Analysis Using Dimethyl Labeling. To examine quantitative changes in the proteome of U937 leukemia cells after shikonin treatment, the dimethyl labeling proteomics analysis was performed. Briefly, $5 \times 10^{6}$ U937 cells were seeded in $10 \mathrm{~mL}$ RPMI 1640 medium in a $25 \mathrm{~cm}^{2}$ culture flask. Subsequently, cells were treated with $0.3 \mu \mathrm{M}$ shikonin (IC50) or DMSO as solvent control for $24 \mathrm{~h}$. After incubation cells were washed with PBS and protein extraction was performed using the M-PER Mammalian Protein Extraction Reagent (Thermo Scientific, Germany) containing a protease inhibitor cocktail (Roche, Germany), cells were incubated with the extraction reagent for $40 \mathrm{~min}$ at $4^{\circ} \mathrm{C}$. After incubation, cellular debris was removed by centrifugation at $14000 \times \mathrm{g}$ for $15 \mathrm{~min}$. Supernatants were transferred into new tubes and an acetone precipitation of the proteins was performed. After precipitation, the acetone was removed and the protein pellet in each of the tube was further diluted to $1 \mu \mathrm{g} / \mu \mathrm{L}$ with $50 \mathrm{mM}$ TEABC and reduced with $5 \mathrm{mM}$ TCEP for $1 \mathrm{~h}$ at $37^{\circ} \mathrm{C}$, followed by alkylation using $2 \mathrm{mM}$ MMTS for $45 \mathrm{~min}$ at room temperature. For the proteolytic digestion, the modified tube-gel digestion protocol was applied and the detergent residue was checked using the method described previously [36]. Two $200 \mu \mathrm{g}$ proteolytic protein mixtures from DMSO and shikonin treated cells were first dissolved in $200 \mu \mathrm{L}$ of $100 \mathrm{mM}$ TEABC ( $\mathrm{pH} \mathrm{8.5)} \mathrm{and,} \mathrm{respectively,}$ mixed with $20 \mu \mathrm{L}$ of formaldehyde and formaldehyde-13C, d2 (4\%, diluted with $\left.\mathrm{H}_{2} \mathrm{O}\right)$. After vortexing (5 min) and centrifugation, each of the sample solutions was mixed with $20 \mu \mathrm{L}$ of $600 \mathrm{mM}$ sodium cyanoborohydride solution. The sample solutions were vortexed $(10 \mathrm{~min})$ and centrifuged again and then allowed to react for $30 \mathrm{~min}$ at $25^{\circ} \mathrm{C}$. To quench the reaction, ammonium hydroxide $(7 \%$ in water, $10 \mu \mathrm{L})$ was added to each sample solution. Finally, $16 \mu \mathrm{L}$ of formic acid was added to acidify each of the sample solutions and two samples were further combined for strong cation exchange (SCX) fractionation.

For SCX fractionation, the buffer SCX-A $\left(5 \mathrm{mM} \mathrm{KH}_{2} \mathrm{PO}_{4}\right.$ in $25 \% \mathrm{ACN}$ at $\mathrm{pH} 3)$ and SCX-B $\left(5 \mathrm{mM} \mathrm{KH} \mathrm{PO}_{4}\right.$ and $350 \mathrm{mM} \mathrm{KCl}$ in $25 \% \mathrm{ACN}$ at $\mathrm{pH} 3$ ) were used as the mobile phase. The peptide mixtures were reconstituted in buffer SCX-A and then loaded into a PolySULFOETHYL A column $(200 \times 2.1 \mathrm{~mm}, 5 \mu \mathrm{m}, 300 \AA$, PolyLC, Columbia, $\mathrm{MD}$ ) for $10 \mathrm{~min}$ at the flow rate of $0.2 \mathrm{~mL} / \mathrm{min}$. Peptides were fractionated using a 75 min gradient from 0 to $100 \%$ of buffer SCX-B. Fractions were collected every three minutes from the retention time of 10 to 55 min using a fraction collector (BioFrac Fraction Collector, BioRad Laboratories, Hercules, CA). The peptide mixtures in each of the fraction were further analyzed by the LC-MS/MS.

LC-MS/MS analysis was performed with a nanoUHPLC system (nanoACQUITY UPLC, Waters, Millford, MA) coupled online to the nanoelectrospray source of a hybrid quadrupole time-of-flight mass spectrometer (Q-TOF-MS) (SYNAPT HDMS G2, Waters, Manchester, UK). For LCMS/MS analysis, water with $0.1 \%$ FA and ACN with $0.1 \%$ FA were used as the mobile phase. The sample was injected into a trap column (Symmetry C18, $5 \mu \mathrm{m}, 180 \mu \mathrm{m} \times 20 \mathrm{~mm}$, Waters, Milford, MA) and separated online with a reverse 
phase column (BEH C18, $1.7 \mu \mathrm{m}, 75 \mu \mathrm{m} \times 250 \mathrm{~mm}$, Waters, Milford, MA) at the flow rate of $300 \mathrm{~nL} / \mathrm{min}$ using either a $95 \mathrm{~min}$ gradient with $5-90 \% \mathrm{ACN} /$ water ratio. The mass spectrometry instruments were all operated in the positive ion mode, and data-dependent acquisition methods were applied for all experiments. The acquisition settings were set to one full MS scan $(350-1600 \mathrm{~m} / \mathrm{z})$ with a scan time of 1 second and switched to six product ion scans $(50-1900 \mathrm{~m} / \mathrm{z})$ with 0.4 second scan time when a precursor ion charge was $2+, 3+$, or $4+$, and the intensity was higher than 200 counts. The data files generated from LC-MS/MS were processed by UniQua and further analyzed by the MASCOT and Trans Proteomics Pipeline (TPP) 1 version 4.4 rev. 1, and the details and criteria of data processing were described previously [37].

4.8. Ingenuity Pathway Analysis. "-omics” datasets were analyzed through the use of ingenuity pathway analysis (IPA, Ingenuity Systems, CA, USA; http://www.ingenuity.com/). This software integrates the experimental results to known biological relationships, mechanisms, and functions using the regularly updated Ingenuity Knowledge Base, a giant database of biological findings and relations gathered from the literature. Only molecules from the "-omics" datasets that met the expression fold change cutoff of $\geq \pm 1.2$ were used for analysis with IPA. Briefly, filtered molecules were fed into Ingenuity Pathway Analysis software, and a separate core analysis was performed for each of the three "-omics" assays to assign the deregulated mRNA, miRNA, and proteins to cellular networks, functions, and pathways. Furthermore, the Ingenuity microRNA Target filter tool was used to associate deregulated miRNAs from the miRNA microarray with experimentally observed and predicted mRNA targets. The results of the core analysis were further studied in a comparison analysis to identify cellular functions affected in all three assays. Finally, the results of the "-omics" assays were merged in one dataset that was screened for overlapping cellular signaling pathways using the core analysis tool. For molecules that showed a nonconsistent deregulation of mRNA and protein expression (36 cases), the stronger deregulated expression values were considered for analysis.

4.9. FlowCellect PI3K-mTOR Signaling Cascade Assay. The FlowCellect PI3K-mTOR signaling cascade assay kit (Merck Millipore, Germany) was used to analyze the effect of shikonin on the PI3K-mTOR signaling pathway. The assay is based on two directly conjugated phospho-specific signaling antibodies against phosphorylated Akt and phosphorylated ribosomal S6 protein, which are both important signaling nodes of the mTOR cascade. The phosphorylation of Akt is indicative of the upstream PI3K signaling, marking the cells initiation into proliferation or cell survival. Phosphorylatedribosomal S6 protein is indicative of downstream mTOR and $\mathrm{p} 70 \mathrm{~S} 6 \mathrm{~K}$ signaling leading to protein translation. The assay was performed according to the manufacture's protocol. Briefly, $1.5 \times 10^{6} \mathrm{U} 937$ cells were treated with $0.3 \mu \mathrm{M}$ shikonin or DMSO solvent control and incubated for $24 \mathrm{~h}$. Subsequently, cells were fixed on ice for 20 min using the supplied fixation buffer. After fixation, cells were washed twice and treated with permeabilization buffer on ice for $20 \mathrm{~min}$. After two further washing steps, cells were resuspended in a final volume of $90 \mu \mathrm{L}$ assay buffer and incubated with $5 \mu \mathrm{L} 20 \mathrm{x}$ anti-phospho-ribosomal protein S6 (Ser235) PerCP conjugated monoclonal antibody and $5 \mu \mathrm{L} 20 \mathrm{x}$ anti-phosphoAkt1/PKB $\alpha$ (Ser473) Alexa Fluor 488 conjugated monoclonal antibody in the dark on ice for one hour. After antibody incubation, cells were centrifuged and resuspended in $500 \mu \mathrm{L}$ assay buffer. Subsequently, cells were measured in a LSRFortessa FACS analyzer (Becton-Dickinson, Germany) and shikonin fluorescence was compensated for the respective channels. For each sample, $1 \times 10^{4}$ cells were counted. The PerCP signal was measured with $488 \mathrm{~nm}$ excitation and detected using a $670 / 30 \mathrm{~nm}$ bandpass filter. The Alexa Fluor 488 signal was analyzed with $488 \mathrm{~nm}$ excitation and detected using a 530/30 nm bandpass filter. All parameters were plotted on a logarithmic scale. Cytographs were analyzed using FlowJo software (Celeza, Switzerland). All experiments were performed at least in triplicate. Student's $t$-test was used for statistical analysis.

4.10. Virtual Screening. Virtual screening represents an approach to identify possible binding interactions between ligands and proteins without any previous knowledge of interactions or binding sites. Since shikonin deregulates the PI3K-mTOR signaling cascade, a virtual screening approach was used to identify proteins of the mTOR pathway which are most likely targeted by shinonin. Virtual screening was performed using AutoDock Vina with the graphical user interface AutoDock Tools [23]. $\mathrm{X}$-ray structures of proteins involved in signaling cascade were downloaded from the "Protein Data Bank" (http://www.pdb.org/). The three-dimensional structure of shikonin was downloaded from the PubChem compound library (http://pubchem.ncbi.nlm.nih.gov/).

4.11. IGF1R Kinase Inhibition Assays. The kinase inhibition assay was performed by ProQinase GmbH (Freiburg, Germany). Shikonin was provided as $1.85 \times 10^{-3} \mathrm{M}$ stock solution in DMSO. In the process, shikonin was serially diluted in semi-log steps with $100 \%$ DMSO in a 96 -well microtiter plate. Directly before use, shikonin was further diluted 1:10 with water. Shikonin was tested at 10 final assay concentrations in the range from $1.85 \times 10^{-5} \mathrm{M}$ to $5.55 \times 10^{-10} \mathrm{M}$. The final DMSO concentration in the reaction cocktails was $1 \%$ in all cases. A radiometric protein kinase assay $\left({ }^{33}\right.$ PanQinase Activity Assay) was used for measuring the kinase activity of IGF1R. All kinase assays were performed in 96-well FlashPlates from Perkin Elmer (Boston, MA, USA) in a $50 \mu \mathrm{L}$ reaction volume. The reaction cocktail was pipetted in 4 steps in the following order: $10 \mu \mathrm{L}$ of nonradioactive ATP solution (in $\mathrm{H}_{2} \mathrm{O}$ ), $25 \mu \mathrm{L}$ of assay buffer/ $\left[\gamma^{33} \mathrm{P}\right]$-ATP mixture, $5 \mu \mathrm{L}$ shikonin in $10 \% \mathrm{DMSO}$, and $10 \mu \mathrm{L}$ of IGF1R/Poly(Glu, Tyr) $4: 1$ mixture. The reaction cocktails were incubated at $30^{\circ} \mathrm{C}$ for 60 minutes. The reaction was stopped with $50 \mu \mathrm{L}$ of $2 \%(\mathrm{v} / \mathrm{v}) \mathrm{H}_{3} \mathrm{PO}_{4}$, and plates were aspirated and washed two times with $200 \mu \mathrm{L} 0.9 \%$ (w/v) $\mathrm{NaCl}$. Incorporation of ${ }^{33} \mathrm{P}_{\mathrm{i}}$ 
(counting of "cpm") was determined with a Wallac MicroBeta scintillation counter (Perkin Elmer). All assays were performed with a Beckman Coulter Biomek 2000/SL robotic system. Kinase activity was evaluated based on a comparison with experiments containing the complete reaction cocktail but no shikonin. A complete reaction cocktail without kinase served as control for unspecific binding of radioactivity to the plate in the absence of protein kinase but in the presence of shikonin. IC50 values were calculated by nonlinear regression using Prism 5.04 (Graphpad, CA, USA).

\section{Abbreviations}

\begin{tabular}{|c|c|}
\hline ACN: & Acetonitrile \\
\hline AML: & Acute myelogenous leukemia \\
\hline AP23573: & Deforolimus \\
\hline APS: & Ammonium persulfate \\
\hline BSA: & Bovine serum albumin \\
\hline CCI-779: & Temsirolimus \\
\hline EGFR: & Epidermal growth factor receptor \\
\hline 4E-BP1: & 4E-binding protein \\
\hline FA: & Formic acid \\
\hline FC: & Fold change \\
\hline HEPES: & $\begin{array}{l}\text { 4-(2-Hydroxyethyl) } \\
\text { piperazine-1-ethanesulfonic acid }\end{array}$ \\
\hline IC50: & Half maximal inhibitory concentration \\
\hline IGF1R: & Insulin-like growth factor 1 receptor \\
\hline IPA: & Ingenuity Pathway Analysis \\
\hline LC-MS: & liquid chromatography-mass spectrometry \\
\hline MMTS: & Methyl methanethiosulfonate \\
\hline MS: & Mass spectrometry \\
\hline mTOR: & Mammalian target of rapamycin \\
\hline mTORC1/2: & $\begin{array}{l}\text { Mammalian target of rapamycin complex } 1 \\
\text { and } 2\end{array}$ \\
\hline PI3K: & Phosphatidylinositol 3-kinase \\
\hline Q-TOF-MS: & Quadrupole time-of-flight mass spectrometer \\
\hline RAD001: & Everolimus \\
\hline RiboS6: & Ribosomal protein S6 \\
\hline ROS: & Reactive oxygen species \\
\hline RTK: & Receptor tyrosine kinase \\
\hline S6K1: & S6 kinase 1 \\
\hline SCX: & Strong cation exchange \\
\hline SDS: & Sodium dodecyl sulfate \\
\hline SGK1: & Serine/threonine-protein kinase Sgk1 \\
\hline TCM: & Traditional Chinese medicine \\
\hline TEABC: & Triethylammonium bicarbonate \\
\hline TCEP: & $\begin{array}{l}\text { Tris (2-carboxyethyl) phosphine } \\
\text { hydrochloride }\end{array}$ \\
\hline TEMED: & $\mathrm{N}, \mathrm{N}, \mathrm{N}^{\prime}, \mathrm{N}^{\prime}$-Tetramethylenediamine \\
\hline TFA: & Trifluoroacetic acid \\
\hline TPP: & Trans-proteomics pipeline \\
\hline TSC1/2: & Tuberous sclerosis proteins 1 and 2 \\
\hline TX-100: & Triton X-100. \\
\hline
\end{tabular}

\section{Conflict of Interests}

The authors declare that there are no significant competing financial, professional, or personal interests that might have influenced the performance or presentation of the work described in this paper.

\section{Acknowledgments}

The authors acknowledge the discussions with Tolga Eichhorn and the molecular dockings of Siegfried Schloissnig. This work was funded by the Landesstiftung RheinlandPfalz; the Institute of Molecular Biology gGmbH (IMB) and the associated Core Facilities are funded by the BoehringerIngelheim Fund. The funders had no role in study design, data collection and analysis, decision to publish, or preparation of the paper.

\section{References}

[1] B. J. Druker, S. Tamura, E. Buchdunger et al., "Effects of a selective inhibitor of the Ab1 tyrosine kinase on the growth of Bcr-Abl positive cells," Nature Medicine, vol. 2, no. 5, pp. 561566, 1996.

[2] D. G. Maloney, A. J. Grillo-López, C. A. White et al., "IDECC2B8 (rituximab) anti-CD20 monoclonal antibody therapy in patients with relapsed low-grade non-Hodgkin's lymphoma," Blood, vol. 90, no. 6, pp. 2188-2195, 1997.

[3] F. Bertolini, "Lenalidomide for multiple myeloma," The New England Journal of Medicine, vol. 367, pp. 573-575, 2012.

[4] W. J. Norde, W. Hobo, R. van der Voort, and H. Dolstra, "Coinhibitory molecules in hematologic malignancies: targets for therapeutic intervention," Blood, vol. 120, pp. 728-736, 2012.

[5] A. M. Martelli, F. Chiarini, C. Evangelisti et al., "Targeting the liver kinase B1/AMP-activated protein kinase pathway as a therapeutic strategy for hematological malignancies," Expert Opinion on Therapeutic Targets, vol. 16, pp. 729-742, 2012.

[6] X. Chen, L. Yang, J. J. Oppenheim, and M. Z. Howard, "Cellular pharmacology studies of shikonin derivatives," Phytotherapy Research, vol. 16, pp. 199-209, 2002.

[7] B. Wiench, T. Eichhorn, M. Paulsen, and T. Efferth, "Shikonin directly targets mitochondria and causes mitochondrial dysfunction in cancer cells," Evidence-Based Complementary and Alternative Medicine, vol. 2012, Article ID 726025, 15 pages, 2012.

[8] T. G. Klenø, B. Kiehr, D. Baunsgaard, and U. G. Sidelmann, "Combination of 'omics' data to investigate the mechanism(s) of hydrazine-induced hepatotoxicity in rats and to identify potential biomarkers," Biomarkers, vol. 9, no. 2, pp. 116-138, 2004.

[9] S. Mizuarai, H. Irie, D. M. Schmatz, and H. Kotani, "Integrated genomic and pharmacological approaches to identify synthetic lethal genes as cancer therapeutic targets," Current Molecular Medicine, vol. 8, no. 8, pp. 774-783, 2008.

[10] F. Meric-Bernstam and A. M. Gonzalez-Angulo, "Targeting the mTOR signaling network for cancer therapy," Journal of Clinical Oncology, vol. 27, pp. 2278-2287, 2009.

[11] G. G. Chiang and R. T. Abraham, "Targeting the mTOR signaling network in cancer," Trends in Molecular Medicine, vol. 13, pp. 433-442, 2007.

[12] C. G. Proud, "mTORC1 signalling and mRNA translation," Biochemical Society Transactions, vol. 37, pp. 227-231, 2009.

[13] A. C. Hsieh, Y. Liu, M. P. Edlind et al., "The translational landscape of mTOR signalling steers cancer initiation and metastasis," Nature, vol. 485, pp. 55-61, 2012. 
[14] N. Chapuis, J. Tamburini, A. S. Green et al., "Perspectives on inhibiting mTOR as a future treatment strategy for hematological malignancies," Leukemia, vol. 24, no. 10, pp. 1686-1699, 2010.

[15] B. Chabner and D. Longo, Cancer Chemotherapy and Biotherapy: Principles and Practice, Lippincott Williams and Wilkins.

[16] A. R. Tee, B. D. Manning, P. P. Roux, L. C. Cantley, and J. Blenis, "Tuberous sclerosis complex gene products, tuberin and Hamartin, control mTOR signaling by acting as a GTPaseactivating protein complex toward Rheb," Current Biology, vol. 13, no. 15, pp. 1259-1268, 2003.

[17] M. Laplante and D. M. Sabatini, "mTOR signaling in growth control and disease," Cell, vol. 149, pp. 274-293, 2012.

[18] E. J. Brown, M. W. Albers, T. B. Shin et al., "A mammalian protein targeted by G1-arresting rapamycin-receptor complex," Nature, vol. 369, pp. 756-758, 1994.

[19] S. Vignot, S. Faivre, D. Aguirre, and E. Raymond, "mTORtargeted therapy of cancer with rapamycin derivatives," Annals of Oncology, vol. 16, pp. 525-537, 2005.

[20] K. E. O’Reilly, F. Rojo, Q. B. She et al., "mTOR inhibition induces upstream receptor tyrosine kinase signaling and activates Akt," Cancer Research, vol. 66, no. 3, pp. 1500-1508, 2006.

[21] J. Tamburini, N. Chapuis, V. Bardet et al., "Mammalian target of rapamycin (mTOR) inhibition activates phosphatidylinositol 3-kinase/Akt by up-regulating insulin-like growth factor1 receptor signaling in acute myeloid leukemia: rationale for therapeutic inhibition of both pathways," Blood, vol. 111, no. 1, pp. 379-382, 2008.

[22] X. Wan, B. Harkavy, N. Shen, P. Grohar, and L. J. Helman, "Rapamycin induces feedback activation of Akt signaling through an IGF-1R-dependent mechanism," Oncogene, vol. 26, no. 13, pp. 1932-1940, 2007.

[23] O. Trott and A. J. Olson, "Software news and update AutoDock Vina: improving the speed and accuracy of docking with a new scoring function, efficient optimization, and multithreading," Journal of Computational Chemistry, vol. 31, no. 2, pp. 455-461, 2010.

[24] F. Singh, D. Gao, M. G. Lebwohl, and H. Wei, "Shikonin modulates cell proliferation by inhibiting epidermal growth factor receptor signaling in human epidermoid carcinoma cells," Cancer Letters, vol. 200, no. 2, pp. 115-121, 2003.

[25] U. Rix and G. Superti-Furga, "Target profiling of small molecules by chemical proteomics," Nature Chemical Biology, vol. 5, pp. 616-624, 2009.

[26] M. Pollak, "The insulin and insulin-like growth factor receptor family in neoplasia: an update," Nature Reviews Cancer, vol. 12, pp. 159-169, 2012.

[27] A. M. Martelli, F. Chiarini, C. Evangelisti et al., "Two hits are better than one: targeting both phosphatidylinositol 3-kinase and mammalian target of rapamycin as a therapeutic strategy for acute leukemia treatment," Oncotarget, vol. 3, pp. 371-394, 2012.

[28] S. Park, N. Chapuis, F. Saint Marcoux et al., "A phase Ib GOELAMS study of the mTOR inhibitor RAD001 in association with chemotherapy for AML patients in first relapse," Leukemia, 2013.

[29] A. M. Martelli, P. L. Tazzari, C. Evangelisti et al., "Targeting the phosphatidylinositol 3-kinase/Akt/mammalian target of rapamycin module for acute myelogenous leukemia therapy: from bench to bedside," Current Medicinal Chemistry, vol. 14, no. 19, pp. 2009-2023, 2007.
[30] D. Barrett, V. I. Brown, S. A. Grupp, and D. T. Teachey, "Targeting the PI3K/AKT/mTOR signaling axis in children with hematologic malignancies," Pediatric Drugs, vol. 14, pp. 299-316, 2012.

[31] S. Amadori, R. Stasi, A. M. Martelli et al., "Temsirolimus, an mTOR inhibitor, in combination with lower-dose clofarabine as salvage therapy for older patients with acute myeloid leukaemia: results of a phase II GIMEMA study (AML-1107)," British Journal of Haematology, vol. 156, pp. 205-212, 2012.

[32] B. Li, S. Gao, F. Wei, A. C. Bellail, C. Hao, and T. Liu, "Simultaneous targeting of EGFR and mTOR inhibits the growth of colorectal carcinoma cells," Oncology Reports, vol. 28, pp. 15-20, 2012.

[33] Y. Sanchez, G. P. Simon, E. Calvino, E. de Blas, and P. Aller, "Curcumin stimulates reactive oxygen species production and potentiates apoptosis induction by the antitumor drugs arsenic trioxide and lonidamine in human myeloid leukemia cell lines," Journal of Pharmacology and Experimental Therapeutics, vol. 335, pp. 114-123, 2010.

[34] J. O’Brien, I. Wilson, T. Orton, and F. Pognan, "Investigation of the Alamar Blue (resazurin) fluorescent dye for the assessment of mammalian cell cytotoxicity," European Journal of Biochemistry, vol. 267, no. 17, pp. 5421-5426, 2000.

[35] J. Eberwine, H. Yeh, K. Miyashiro et al., "Analysis of gene expression in single live neurons," Proceedings of the National Academy of Sciences of the United States of America, vol. 89, no. 7, pp. 3010-3014, 1992.

[36] C. J. Chen, M. C. Tseng, H. J. Lin, T. W. Lin, and Y. R. Chen, "Visual indicator for surfactant abundance in MS-based membrane and general proteomics applications," Analytical Chemistry, vol. 82, pp. 8283-8290, 2010.

[37] W. H. Chang, C. Y. Lee, C. Y. Lin et al., "UniQua: a universal signal processor for MS-based qualitative and quantitative proteomics applications," Analytical Chemistry, vol. 85, pp. 890$897,2013$. 


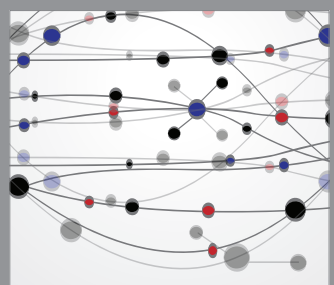

The Scientific World Journal
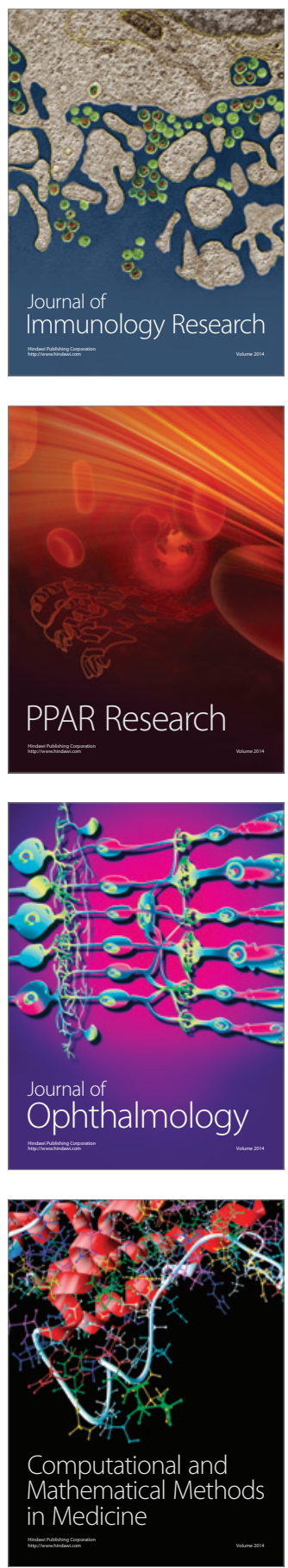

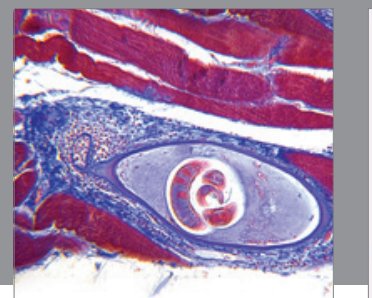

Gastroenterology

Research and Practice
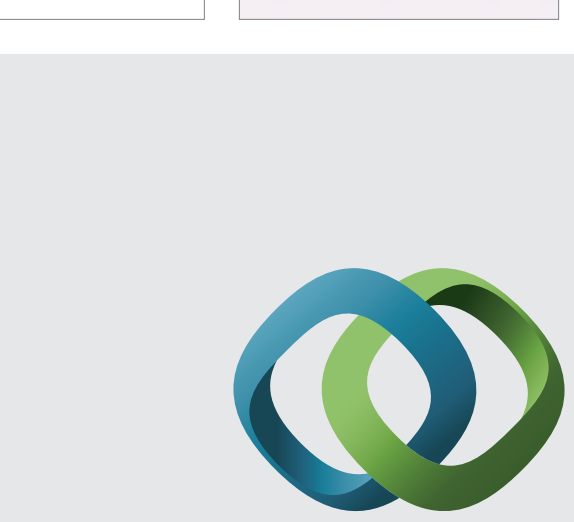

\section{Hindawi}

Submit your manuscripts at

http://www.hindawi.com
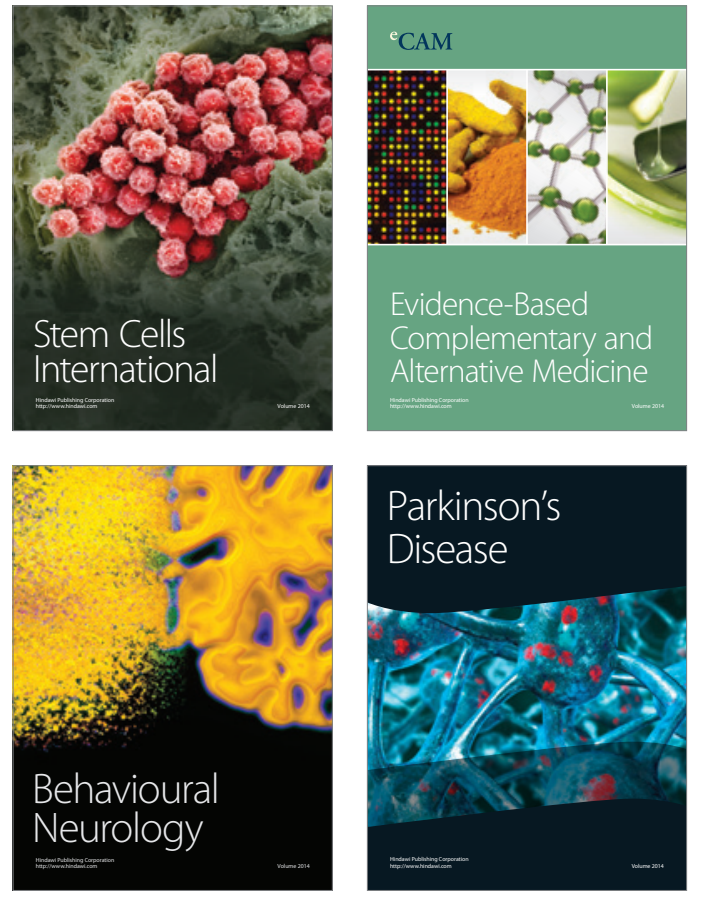
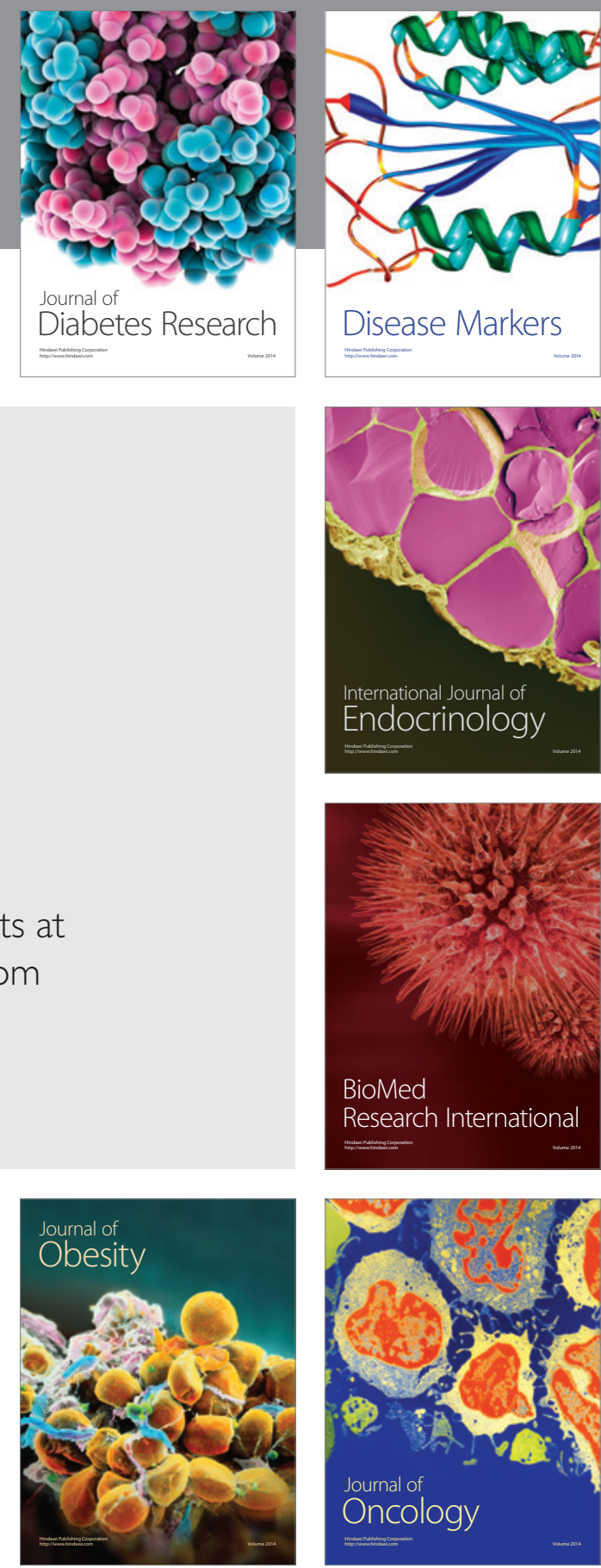

Disease Markers
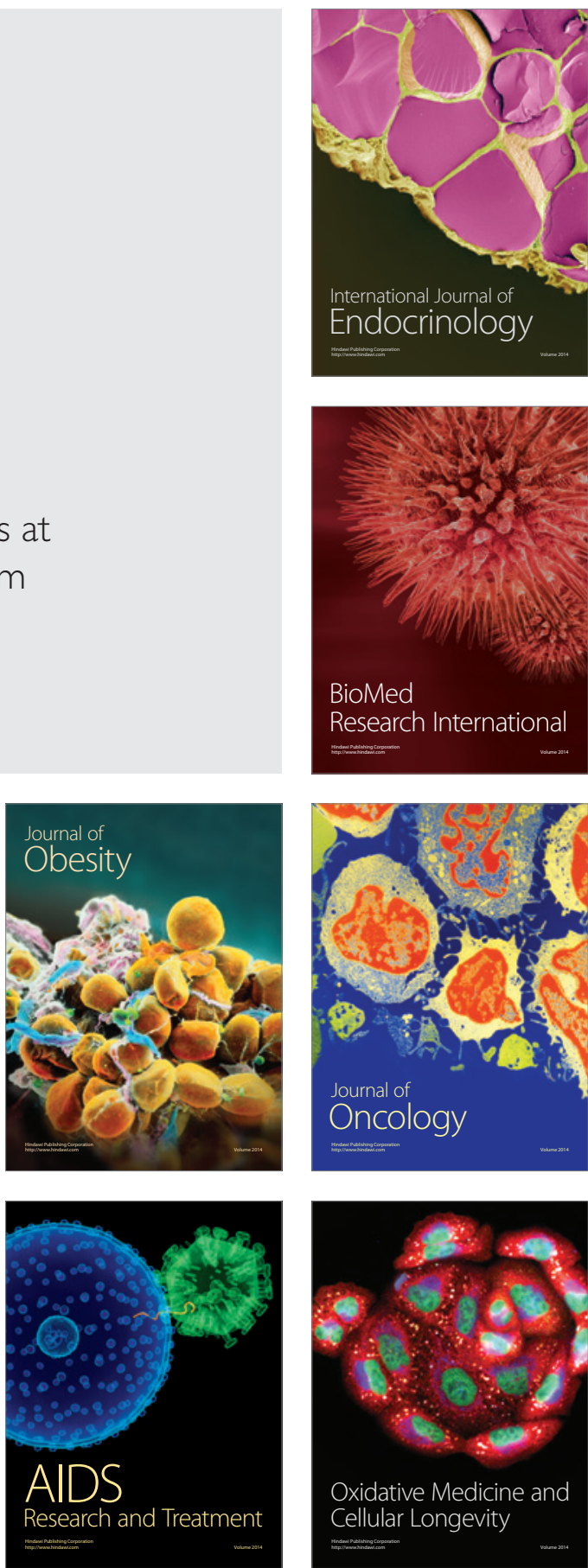